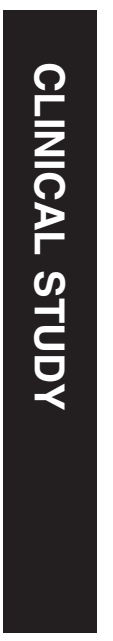

\title{
Partial posterior hyaloidectomy for macular disorders
}

'Department of Ophthalmology, Kim's Eye Hospital, Myung-Gok Eye Research Institute, Konyang University College of Medicine, Seoul, Korea

2Department of Ophthalmology, Samsung Medical Center, Sungkyunkwan University School of Medicine, Seoul, Korea

${ }^{3}$ The Institute of Ophthalmology and Optometry, Department of Ophthalmology, School of Medicine, Ewha Women's University, Seoul, Korea

Correspondence:

SW Kang, Department of Ophthalmology, Samsung Medical Center, Sungkyunkwan University School of Medicine, 50 Irwon-dong, Gangnam-gu, Seoul 135-710, Korea. Tel: +82 234103562 ; Fax: +82 234100074 .

E-mail: swkang@skku.edu

Meeting presentation: This study was presented at the 105th Annual Meeting of Korean Ophthalmological Society, April 2011, Busan, Korea.

Received: 9 January 2013 Accepted in revised form: 16 April 2013 Published online: 7 June 2013

\begin{abstract}
Purpose To evaluate the effect of partial posterior hyaloidectomy (PPH) in preventing iatrogenic retinal breaks related to the induction of a posterior vitreous detachment (IPVD)

Methods Fifty-nine patients who necessitated IPVD for an epiretinal membrane or macular hole were included in this prospective, interventional case series. Extensive removal of vitreous gel, close to the retina, was conducted before IPVD under $23 \mathrm{G}$ (gauge)-vitrectomy system. The PPH involved the limited extent of IPVD and limited removal of the outermost vitreous cortex to an area slightly beyond the margin of the temporal major vascular arcade. The incidence of retinal breaks related to the surgery was compared with 57 eyes that had undergone conventional 23-G total vitrectomy accompanied by extensive IPVD using $\chi^{2}$-test. Results Patients were followed-up for a mean of 14.3 months (6-30 months) after the surgery. The incidence of peripheral retinal breaks after the PPH was 3.4\% (2/59 eyes), which was significantly lower than that in the eyes that underwent conventional $23 \mathrm{G}$ vitrectomy $(15.8 \%, 9 / 57$ eyes, $P=0.023)$ for the same disorders that required an IPVD. No patient complained of postoperative floaters, postoperatively.

Conclusions PPH would be an efficient procedure to prevent iatrogenic peripheral retinal breaks related to an IPVD.

Eye (2013) 27, 946-951; doi:10.1038/eye.2013.117; published online 7 June 2013
\end{abstract}

Keywords: partial posterior hyaloidectomy; iatrogenic retinal break; posterior vitreous detachment; macular hole; epiretinal membrane

\section{Introduction}

Iatrogenic retinal breaks are known to be a causative element of rhegmatogenous retinal detachment, one of the most serious sightthreatening complications of pars plana vitrectomy. The induction of a posterior vitreous detachment (IPVD) during vitrectomy has been suggested as one of the etiologies of retinal breaks. ${ }^{1-4}$ In particular, this is suspected to be the main cause when the break is located further away from the sclerotomy site.

Previous studies that investigated the difference in the incidence of surgery-related retinal breaks between eyes with and without an IPVD demonstrated that the incidence in eyes with IPVD (12.4-21.1\%) was much higher than that in eyes without an IPVD $(1.4-8.4 \%),{ }^{1,3-5}$ However, a fundamental strategy to prevent the development of iatrogenic retinal breaks related to an IPVD has not yet been established. Some investigators have recommended a careful intraoperative and postoperative peripheral examination at the end of each vitrectomy. $1,3,4,6$ More detailed techniques such as scleral depression ${ }^{1,4}$ and use of a wide-angle viewing system ${ }^{4}$ have been recommended to minimize the incidence of a missed break. However, these recommendations were focused mainly on early detection and treatment of breaks rather than preventing the development of the break itself. Although other investigators have recommended avoiding vitreous base traction when inducing and extending a PVD ${ }^{1}$ and more tangential stripping of the vitreous cortex to the retina, ${ }^{2}$ the effect of these methods has not yet been addressed.

The iatrogenic retinal breaks related to an IPVD usually develop near or anterior to the equatorial region. ${ }^{2,4}$ Thus, we hypothesized that this complication could be prevented by restricting the extent of the IPVD. We planned a modified vitrectomy procedure in which the extent of the IPVD and removal of vitreous cortex was restricted to an area slightly beyond the margin of the temporal major vascular arcade. We have termed this procedure as partial posterior hyaloidectomy (PPH). Unlike 
retinal detachment or proliferative diabetic retinopathy, lesions of macular disorders, such as macular hole (MH) or epiretinal membrane (ERM), are localized within the major vascular arcade. Therefore, these macular disorders could be a good candidate for this procedure.

The purpose of the current study was to evaluate the effect of a PPH on preventing iatrogenic retinal breaks related to an IPVD in the treatment of a MH and ERM.

\section{Materials and methods}

This prospective, interventional, case series was performed at a single center according to the tenets of the Declaration of Helsinki. The study was approved by the institutional review board (Samsung Medical Center IRB no. 2011-05-011), and written informed consent was obtained from all patients before enrollment. The PPH was prospectively planned, whereas data of control group were collected via retrospective review of medical records.

\section{Patients}

From November 2009 through January 2012, PPH was prospectively planned for 68 patients with idiopathic ERM or idiopathic MH without evidence of a PVD preoperatively. The presence of a Weiss ring was regarded as one of the important preoperative evidence of a PVD. However, the ultimate confirmation of a PVD was made based on intraoperative findings. Only eyes in which a PVD was induced during surgery were included in the study. Any cases involving ocular inflammation, diabetic retinopathy, retinal vasculitis, and refractive errors of more than $-6.0 \mathrm{D}$ (diopters) were excluded. Cases that developed after trauma were also excluded. Each patient underwent a complete preoperative evaluation, including an examination for best-corrected Snellen visual acuity (BCVA), anterior segment examination, and dilated fundus examination with a 90-D lens. An indirect ophthalmoscopic examination of the peripheral retina with a scleral indentation was conducted before surgery in all eyes.

\section{Surgical technique}

A three-port pars plana vitrectomy using a 23-G (gauge) vitrectomy system, either Associate (Dutch Ophthalmic Research Center Inc., Zuidland, The Netherlands) or Accurus (Alcon Laboratories Inc., Fort Worth, TX, USA), with a one-step incision ${ }^{7}$ was conducted by one righthanded surgeon (SWK). An infusion cannula was positioned in the inferotemporal quadrant, and superotemporal and superonasal sclerotomies were placed near the 10- and 2-o'clock meridians. The peripheral fundus was visualized using a wide-angle viewing system.

The core vitrectomy in a PPH entails more extensive removal of the vitreous gel than in the conventional core vitrectomy (Figure 1). In this procedure, as much of the vitreous gel as possible was removed before the IPVD. During the core vitrectomy, the vacuum was set at $500 \mathrm{~mm} \mathrm{Hg}$ and the cut rate was $2500 \mathrm{cuts} / \mathrm{min}$. Removal of peripheral vitreous was performed by using $\sim 300 \mathrm{~mm} \mathrm{Hg}$ of vacuum with the same cutting rate. The IPVD was conducted by engaging the attached posterior cortical vitreous with a $23-\mathrm{G}$ needle with the angulated tip in the area adjacent to the optic disc followed by gently moving the tip to approximately three disc diameters away from the margin of the optic disc. Then, the tip was slightly elevated in a posteroanterior direction and slowly advanced to the extent planned. With the IPVD, the surgeon was able to visualize a floating Weiss ring in all of the cases. In the temporal direction, the extension of the PVD was restricted to approximately two disc diameters distance beyond the margin of the temporal major vascular arcade. The operator then removed the outermost vitreous cortex within the extent of the PVD (Figure 2), maintaining the position of the vitreous cutter less than two disc diameters away from the retinal surface. The vacuum was usually lowered to the $200 \mathrm{~mm} \mathrm{Hg}$ range and the cut rate was 2500 cuts $/ \mathrm{min}$. At the end of this procedure, only a thin vitreous shell remained (Figure 1).

To minimize floater symptoms, which could develop postoperatively due to inferior deflection of the residual superior vitreous by gravity in an upright position, the extent of outermost cortical vitreous removal was enlarged slightly to superior side.

After vitreous cortex removal, ERM and internal limiting membrane removal were performed. Before the conclusion of vitreous surgery and before fluid-air exchange in the eyes with $\mathrm{MH}$, the peripheral retina was examined with scleral depression under a wide-angle viewing system.

Follow-up examinations were scheduled approximately once every 1-4 weeks after the surgery during the first 3 months, and then once every 2-3 months according the patient's status. At every visit, the presence of floaters was documented and careful peripheral retinal examination using a 90-D lens and/or an indirect ophthalmoscope was performed.

\section{Outcome measures}

The patients that completed a follow-up of at least 6 months were included in the analysis. The incidence of retinal breaks related to the surgery and postoperative complaints of floaters were evaluated. The incidence of 
a

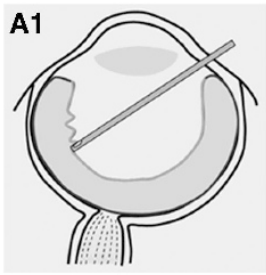

b

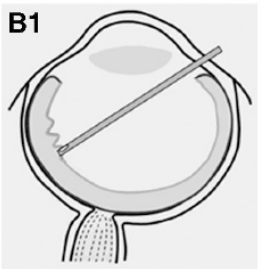

Conventional vitrectomy
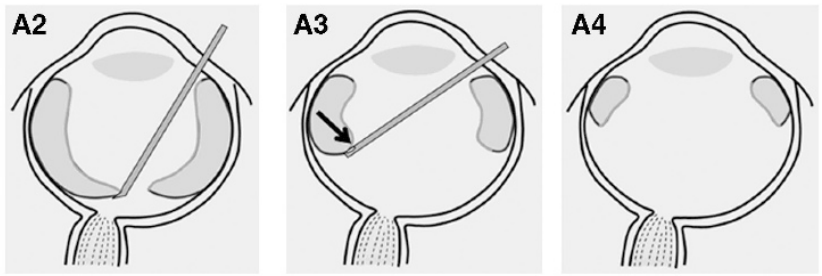

Partial posterior vitrectomy
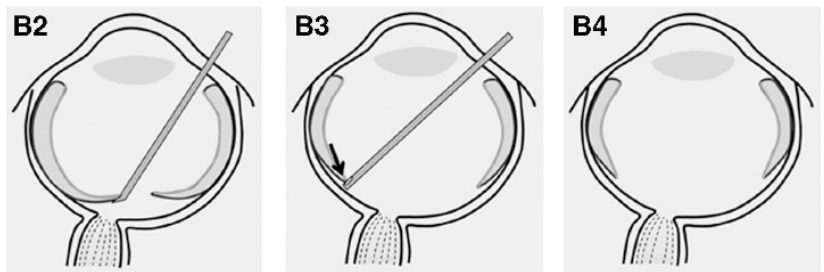

Figure 1 A diagram illustrating the conventional vitrectomy (a) and PPH procedures (b). Each pair of pictures shows the difference in specific techniques between the two surgeries. A1, B1: core vitrectomy; A2, B2: IPVD utilizing a bent needle; A3, B3: vitreous cortex removal; A4, B4: residual vitreous and the extent of posterior vitreous detachment after the surgery. In a $\mathrm{PPH}$ procedure, as much of the vitreous gel as possible is removed before the IPVD. Notice the thin residual vitreous cortex after core vitrectomy (B1). After the IPVD, the extent of vitrectomy was restricted to just beyond the margin of the temporal major vascular arcade to avoid peripheral propagation of the PVD. In addition, an attempt to minimize the perpendicular traction to the vitreous base was made by maintaining the position of the vitreous cutter very close to the retinal surface, and by lowering the suction power (B3). Arrows indicate the expected direction of traction force during vitreous cortex removal (A3, B3).

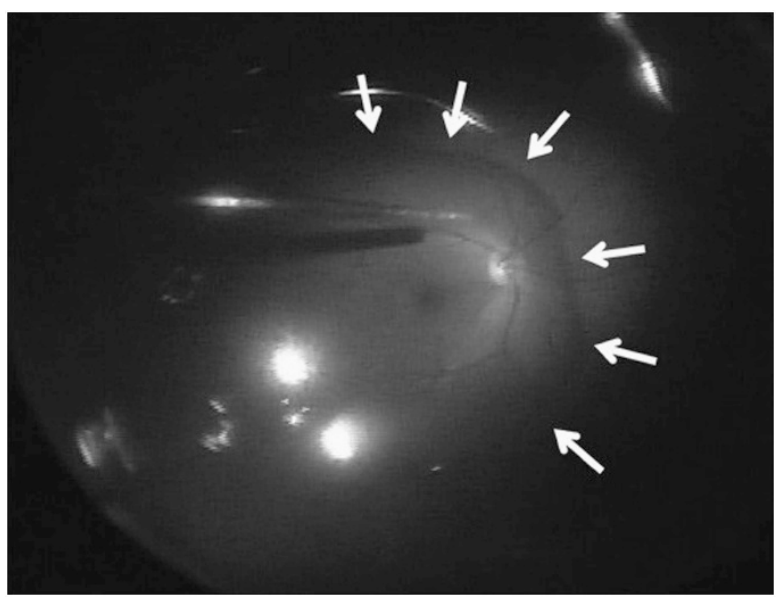

Figure 2 An intraoperative capture image taken during the fluid-air exchange in an eye with $\mathrm{MH}$. It shows the extent of IPVD and removal of vitreous cortex in PPH. White arrows indicate margin of residual vitreous cortex.

retinal breaks related to the surgery was compared with that in the 57 eyes of 57 patients who were diagnosed with either an ERM or $\mathrm{MH}$, and underwent both extensive IPVD and total vitrectomy using a 23-G system from July 2008 through October 2009. Data of these 57 eyes were collected via retrospective review of medical records. All the vitrectomy was also performed by the same surgeon (SWK). Only patients who satisfied the inclusion and exclusion criteria of the current study were included. In addition, the incidence or retinal breaks according to utilizing different vitrectomy systems, that were Accurus and Associate, was estimated.

\section{Statistics}

Statistical analysis was performed using a commercially available statistical package (SPSS ver. 18.0 for Windows; SPSS Inc., Chicago, IL, USA). Baseline characteristics were compared between groups using an independent samples $t$-test and the $\chi^{2}$-test. The incidence of retinal breaks related to the surgery was compared between the two groups using both the $\chi^{2}$-test and Log-rank test. The incidences of retinal breaks related to different vitrectomy systems were compared using Fisher's exact test.

\section{Results}

Fifty-nine of 68 patients (59 eyes, 19 ERMs, and 40 MHs) were followed for 6 months or more. The mean follow-up period was $14.3 \pm 5.9$ months (range 6-30 months).

Combined phacoemulsification and posterior chamber intraocular lens implantation was performed in 9 (18.4\%) out of 49 phakic eyes. The baseline characteristics recorded for the patients are summarized in Table 1. The preoperative BCVAs (logarithm of the minimum angle of resolution units; mean \pm SD) were $0.49 \pm 0.29$, and improved to $0.29 \pm 0.24$ at the final visit. 
Table 1 Clinical characteristics of patients and the differences in the incidence of retinal breaks related to the surgery among the PPH group and conventional vitrectomy group

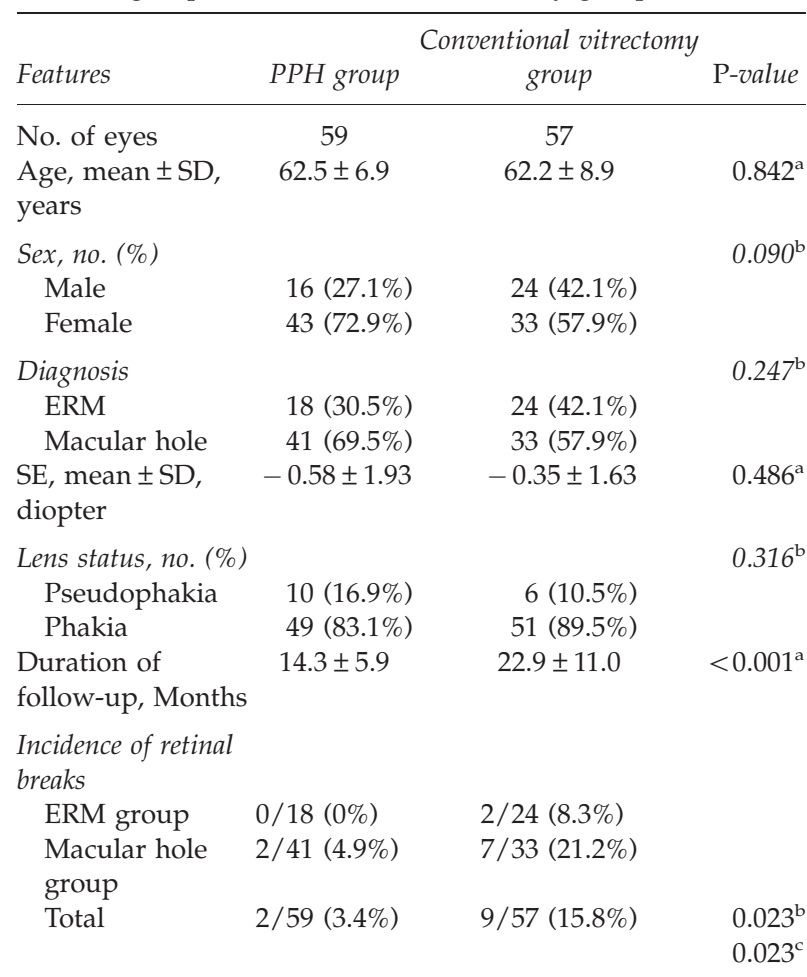

Abbreviations: ERM, epiretinal membrane; PPH, partial posterior hyaloidectomy; SE, spherical equivalent.

All eyes in the two groups underwent induction of a posterior vitreous detachment during the vitrectomy.

${ }^{a}$ Independent samples $t$-test.

${ }^{\mathrm{b}} \chi^{2}$-test.

${ }^{\mathrm{c}}$ Log-rank test.

\section{Incidence of retinal breaks}

Three retinal breaks were identified in three eyes in the preoperative examination and were treated with endolaser photocoagulation. Intraoperative development of retinal break was not noted in any eye. Retinal breaks related to surgery were found in two eyes (3.4\%) during the postoperative examination. Postoperative retinal detachment was detected in two eyes that had been operated on for $\mathrm{MH}$. In one eye, two retinal breaks, which were located at the 9:30 and 12:30 meridians of the peripheral retina anterior to the equator were identified 4 months after the surgery and treated successfully with a scleral buckling procedure. In the other eye, one retinal break, which was located at the 6:30 meridian of the peripheral retina, was identified 2 days after the surgery and treated successfully with a scleral buckling procedure.

Table 1 shows baseline characteristics of the PPH group and the conventional 23 G-vitrectomy group. All the eyes in the two groups were suffered with surgical induction of PVD during vitrectomy for either an ERM or $\mathrm{MH}$. Fifty-seven eyes of 57 patients were included in the conventional $23 \mathrm{G}$-vitrectomy group (24 ERMs and 33 MHs). Twelve retinal breaks related to the surgery were verified in nine eyes; an incidence of $15.8 \%$ (9/57 eyes). Retinal breaks were identified during the surgery in 7 eyes. In two eyes, retinal breaks were detected within 3 weeks postoperatively. The incidence of retinal breaks related to the surgery following PPH was significantly lower than that in the conventional $23 \mathrm{G}$-vitrectomy group in both $\chi^{2}$-test (Table $1, P=0.023$ ) and log-rank test (Table $1, P=0.023)$. The distribution of retinal breaks in the two groups is shown in Figure 3.

The peristaltic pump was used when vitrectomy was performed with Associate system. Accurus was used in 37 eyes (24 eyes in PPH group and 13 eyes in conventional $23 \mathrm{G}$-vitrectomy group) and retinal breaks related to the surgery were developed in 2 eyes (5.4\%). Associate was used in 79 eyes (35 eyes in PPH group and 44 eyes in conventional 23G-vitrectomy group) and the retinal breaks was developed in 9 eyes (11.4\%). The difference in the incidence between two vitrectomy systems was not significant (Fisher's exact test; $P=0.165)$.

\section{Operative or postoperative complications other than retinal breaks in PPH group}

Minimal bleeding from the retinal vessel occurred in one eye $(1.7 \%)$ and was treated by increasing the height of the fluid bottle. Four eyes (6.8\%) presented with an intraocular pressure (IOP) $<6 \mathrm{~mm} \mathrm{Hg}$ on postoperative day 1 , and one also developed a shallow choroidal effusion. The hypotony and choroidal effusion resolved spontaneously within a week. There was no postoperative sclerotomy leakage requiring sutures. Two eyes $(3.4 \%)$ had an increased IOP $>23 \mathrm{~mm} \mathrm{Hg}$ after the surgery, and were successfully treated with antiglaucoma medications. Fourteen eyes underwent cataract surgery during the follow-up period. No patient complained of floaters during the study period.

\section{Discussion}

The primary origin of iatrogenic retinal breaks related to an IPVD may be a strong artificial traction force on the retina exerted by the surgical manipulation. We think that there are two sources of this traction. One source is the IPVD itself. The traction force is applied to the retina when the posterior cortical vitreous is pulled anteriorly. ${ }^{2}$ The other possible source of traction is when the vitreous cortex is removed near the vitreous base in which a firm vitreo-retinal adhesion is expected. Given the position and direction of the vitreous cutter in conventional 
vitrectomy, some degree of perpendicular traction to the vitreous base cannot be avoided (Figure 1-A3).

Considering the similar propensity for the incidence of PVD-related retinal break in macular surgery despite the evolution of the vitrectomy machine, $, 1,3,4,6,8-12$ the effect of increasing cut velocity may be limited. Some degree of artificial vitreous traction seems to be inevitable during peripheral vitreous removal.

The primary goal of the PPH technique is avoiding these two sources of traction. To avoid the former source of traction, we restricted the extent of the manual IPVD. It is possible that some extension of a PVD may occur during the vitrectomy because of traction from the vitreous cutter, although most of the procedures are performed far from the vitreous base. However, we are sure that this traction will be much weaker than that exerted by a manual IPVD. Slow postoperative extension of a PVD may also occur. As suggested in a report by Tabandeh $e t ~ a l, 2$ postoperative breaks may develop during the extension of an incomplete PVD. However, because the traction force related to the postoperative extension of a PVD is thought to be much weaker than the artificial traction force exerted by surgical manipulation, the risk of developing a retinal break by the extension of an incomplete PVD will not exceed its natural incidence.

To avoid the latter source of traction, we planned three strategies. First, as much vitreous gel as possible was removed near the vitreous base before the IPVD. Although the vitreous cutter approached the retina during the removal of vitreous gel near the vitreous base, the traction force to the focal retinal area was assumed to be very weak without the presence of a PVD. However, after the IPVD, the removal of the outermost vitreous cortex was limited to the posterior pole, far from the vitreous base. Second, the position and angle of the vitreous cutter was carefully manipulated to avoid perpendicular traction to the retina. To achieve tangential rather than perpendicular traction to the peripheral retina during removal of the outermost vitreous cortex in the posterior pole, the vitreous cutter had to approach the retina as closely as possible (Figure 1-B3). Lastly, the suction power was reduced to less than half of the usual setting used in a conventional vitrectomy. As a result, the incidence of retinal breaks related to a PPH was significantly lower than that found in patients who had undergone an extensive IPVD and total vitrectomy (conventional vitrectomy group).

The mean follow-up period after PPH was shorter than that of the conventional $23 \mathrm{G}$-vitrectomy group.

Considering the finding that all the retinal breaks related to surgery in both the PPH group and the conventional $23 \mathrm{G}$-vitrectomy group were identified within 5 months after surgery, we think that a difference in follow-up

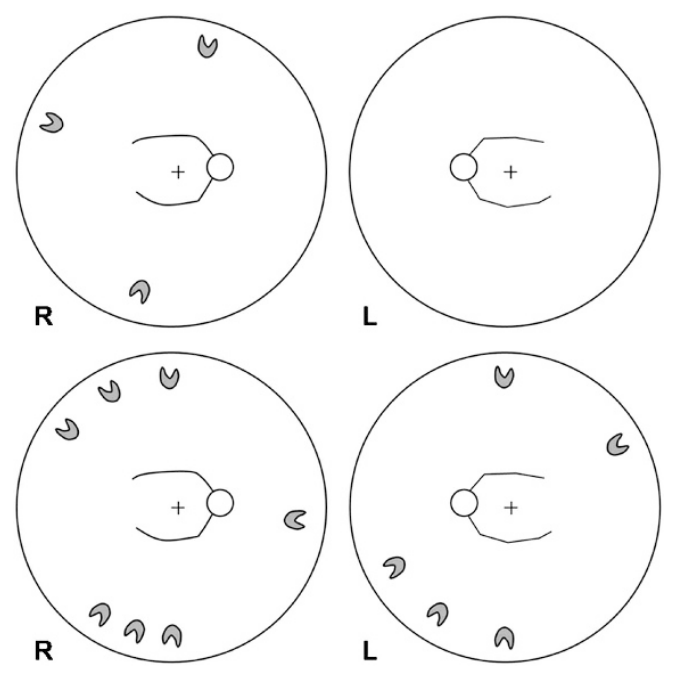

Figure 3 Diagrams showing the distribution of retinal breaks from two eyes in the PPH group (top) and from nine eyes in the conventional $23 \mathrm{G}$-vitrectomy group (bottom).

period between the two groups may not have a significant influence on the result of this study.

The locations of the retinal breaks that developed in the PPH group are noteworthy. The distribution of retinal breaks differs according to etiology. The breaks located in the superior quadrants, particularly in the quadrant near the surgeon's dominant hand, are considered sclerotomy-related breaks. ${ }^{1,3,6,12,13}$ On the other hand, an IPVD is thought to be an important etiology when the breaks are located in the inferior quadrants. ${ }^{2,3,5}$ Two of three $(66.7 \%)$ retinal breaks in the PPH group were identified in the superior quadrants, near the sclerotomy sites, suggesting the etiology of these breaks could be sclerotomy-related rather than IPVD-related.

Although specific data were not presented in this study, we believe the another advantage of a PPH is that the operation time can be reduced by skipping the extensive peripheral vitreous removal after the IPVD, and by reducing the possible need for endolaser photocoagulation for the treatment of iatrogenic peripheral retinal break.

One possible side-effect of a PPH would be a postoperative complaint of floaters. In particular, possible inferior deflection of superior residual vitreous due to gravity may exacerbate this problem. Thus, we tried to minimize this complication by removing more vitreous superiorly than inferiorly after the IPVD. None of our patients reported symptomatic floaters during the follow-up period.

This study has several limitations, which include non-randomized nature of the study, use of retrospective control group, and potential selection bias by excluding myopic cases. In addition, the conclusion was made 
based on the single-center experience with relatively short follow-up period.

In summary, $\mathrm{PPH}$ was found to be beneficial with regard to preventing iatrogenic retinal breaks related to IPVD in our series. We hope that, based on long-term follow-up results, further adjustments to this modified vitrectomy procedure may contribute toward establishing a safer and a more efficient vitrectomy technique for macular disorders necessitating an IPVD.

\section{Summary}

What was known before

- The induction of a posterior vitreous detachment during vitrectomy has been suggested as one of the etiologies of retinal breaks.

What this study adds

- Partial posterior hyaloidectomy would be an efficient procedure to prevent iatrogenic peripheral retinal breaks related to an induction of a posterior vitreous detachment.

\section{Conflict of interest}

The authors declare no conflict of interest.

\section{References}

1 Ramkissoon YD, Aslam SA, Shah SP, Wong SC, Sullivan PM. Risk of iatrogenic peripheral retinal breaks in 20-G pars plana vitrectomy. Ophthalmology 2010; 117: 1825-1830.

2 Tabandeh H, Chaudhry NA, Smiddy WE. Retinal detachment associated with macular hole surgery: characteristics, mechanism, and outcomes. Retina 1999; 19: 281-286.

3 Tan HS, Mura M, de Smet MD. Iatrogenic retinal breaks in 25-gauge macular surgery. Am J Ophthalmol 2009; 148: 427-430.

4 Chung SE, Kim KH, Kang SW. Retinal breaks associated with the induction of posterior vitreous detachment. Am J Ophthalmol 2009; 147: 1012-1016.

5 Sandali O, El Sanharawi M, Lecuen N, Barale PO, Bonnel S, Basli E et al. 25-, 23-, and 20-gauge vitrectomy in epiretinal membrane surgery: a comparative study of 553 cases. Graefes Arch Clin Exp Ophthalmol 2011; 249: 1811-1819.

6 Guillaubey A, Malvitte L, Lafontaine PO, Hubert I, Bron A, Berrod JP et al. Incidence of retinal detachment after macular surgery: a retrospective study of 634 cases. Br J Ophthalmol 2007; 91: 1327-1330.

7 Eckardt C. Transconjunctival sutureless 23-gauge vitrectomy. Retina 2005; 25: 208-211.

8 de Bustros S, Thompson JT, Michels RG, Rice TA, Glaser BM. Vitrectomy for idiopathic epiretinal membranes causing macular pucker. Br J Ophthalmol 1988; 72: 692-695.

9 Grewing R, Mester U. Results of surgery for epiretinal membranes and their recurrences. Br J Ophthalmol 1996; 80: 323-326.

10 Michels RG. Vitrectomy for macular pucker. Ophthalmology 1984; 91: 1384-1388.

11 Hikichi T, Matsumoto N, Ohtsuka H, Higuchi M, Matsushita $\mathrm{T}$, Ariga $\mathrm{H}$ et al. Comparison of one-year outcomes between 23- and 20-gauge vitrectomy for preretinal membrane. Am J Ophthalmol 2009; 147: 639-643; e631.

12 Moore JK, Kitchens JW, Smiddy WE, Mavrofrides EC, Gregorio G. Retinal breaks observed during pars plana vitrectomy. Am J Ophthalmol 2007; 144: 32-36.

13 Oyakawa RT, Schachat AP, Michels RG, Rice TA. Complications of vitreous surgery for diabetic retinopathy. I. Intraoperative complications. Ophthalmology 1983; 90: 517-521. 